\title{
The effect of the surface distribution of elements on measuring the magnetic field of chemically peculiar stars ${ }^{\star}$
}

\section{The case of the roAp star HD 24712}

\author{
F. Leone and G. Catanzaro
}

\author{
INAF - Osservatorio Astrofisico di Catania, via S. Sofia 78, 95123 Catania, Italy \\ e-mail: [fleone; gcatanzaro]@ct.astro.it
}

Received 2 February 2004 / Accepted 4 June 2004

\begin{abstract}
A non-homogeneous distribution over the stellar surface of the chemical elements and the presence of a large-scale magnetic field are invoked in order to explain the periodic line strength, photometric and magnetic variability of magnetic chemically peculiar stars. In such a framework, the variability period is identical to the rotational period.

In magnetic chemically peculiar stars, the so-called effective magnetic field $H_{\text {eff }}$, the average over the visible stellar disk of the longitudinal field component weighted by the local line strength, is routinely measured from Stokes $I$ and $V$ profiles of selected spectral lines. In spite of evidence that the distribution of the various chemical elements over the stellar surface can be inhomogeneous and different from element to element, $H_{\text {eff }}$ values obtained from lines of different elements are often statistically combined to improve the accuracy of effective field measurements. Similarly, mean high $S / N$ profiles are obtained from the profiles of lines of different elements.

We have established, by means of $R=115000$ circular spectropolarimetry of the magnetic chemically peculiar star HD 24712 in the 4700-7000 $\AA$ range, the dependence of the measurements of $H_{\text {eff }}$ on the atomic weight of 24 elements (from carbon to erbium, if possible at different ionisation states). At all 3 rotational phases considered, $H_{\text {eff }}$ values derived from different elements can differ by up to $800 \mathrm{G}$. We find an overall increase in $H_{\text {eff }}$ with atomic number and a maximum near $Z=60$. The behaviour of sodium is quite singular inasmuch as it always exhibits a negative value of the field, peaking at $-0.39 \mathrm{kG}$ when the rare earths give $H_{\mathrm{eff}}=1.2 \mathrm{kG}$. Under the assumption of a dipolar field, we conclude that the elements giving the largest values of $H_{\mathrm{eff}}$ are concentrated near the positive polar region and that the other elements are more homogeneously distributed over the stellar surface or concentrated in belts around the magnetic equator. Sodium seems to be localised in the negative magnetic hemisphere only.

This picture is corroborated by the equivalent width variability of the lines: up to iron, equivalent widths changes out-of-phase with respect to the $H_{\text {eff }}$ variability; elements heavier than iron present equivalent widths that are variable in-phase. We do not find any relation between the respective amplitudes of the equivalent width variations and the atomic numbers of the elements. For iron, the observed equivalent width variability does not seem to be simply related to any non-homogeneous distribution over the stellar surface.

We conclude that measurements originating from different elements cannot in general be combined to improve the precision of $H_{\text {eff }}$ measurements. Indeed, any modelling attempt based on the periodic variations in $H_{\text {eff }}$ is subject to the risk that the sampling of the magnetic field over the stellar surface by the lines of a given chemical element is uneven or incomplete.
\end{abstract}

Key words. stars: magnetic fields - stars: chemically peculiar - stars: individual: HD 24712

\section{Introduction}

The magnetic chemically peculiar stars (hereafter CP2 stars according to Preston's 1974 nomenclature) of the upper main sequence present photometric, spectral and magnetic periodic variability. For this class of stars, it is commonly accepted that the stellar surface is characterised by the presence of a non-homogeneous distribution of various chemical elements and a magnetic field organised on a large scale. Thus the

\footnotetext{
^ In memory of Horace Babcock.
}

above-mentioned periodic variations are a consequence of the stellar rotation (Babcock 1949a). Starting with Deutsch (1958), much effort has been devoted to the mapping of the distributions of the elements and it is now evident that these nonhomogeneous distributions can be very different for the various elements (Kuschnig et al. 1999) and even for the various ionisation states of an element (Strasser et al. 2001).

By means of circular spectropolarimetry (Babcock 1947), it is possible to measure the effective magnetic field of CP2 stars, i.e. the line-intensity weighted average over the visible stellar 
disk of the line-of-sight component $H_{\mathrm{z}}$ of the magnetic field vector (Mathys 1991):

$$
\begin{aligned}
H_{\mathrm{eff}}= & \frac{1}{W \mathcal{F}_{I_{\mathrm{c}}}} \int_{0}^{2 \pi} \mathrm{d} \phi \int_{0}^{\pi / 2} H_{\mathrm{z}} \cos \theta \sin \theta \mathrm{d} \theta \\
& \times \int\left[I_{\mathrm{c}}-I_{\lambda}\right] \mathrm{d} \lambda
\end{aligned}
$$

where $W$ is the line equivalent width, $\mathcal{F}_{I_{\mathrm{c}}}$ is the flux in the continuum at the line wavelength, $\phi$ and $\theta$ define in the usual way the coordinates on the stellar surface. $I_{\mathrm{c}}$ and $I_{\lambda}$ are the respective intensities in the continuum and in the line at the point $(\theta, \phi)$.

Usually, $H_{\text {eff }}$ values obtained from lines of different elements are statistically combined with the aim to improve the precision of the effective field measurement. However, because of the non-homogeneous distribution of the elements, such an operation is not at priori expected to lead to any improvement and moreover its validity has yet to be proved.

The determination of the magnetic field geometry of CP2 stars is often based on the modelling of the rotational modulation of $H_{\text {eff }}$ (Preston 1972; Stift 1975; Bonsack 1979; Landstreet \& Mathys 2000). If elements used to measure $H_{\text {eff }}$ are not homogeneously distributed over the stellar surface, the magnetic geometries thus determined could be biased due to a weighted or incomplete sampling of the stellar visible disk.

We have decided to quantify the importance of the nonhomogeneous distribution of elements in measuring $H_{\text {eff }}$, and have to this purpose carried out $R=115000$ circular spectropolarimetric observations of HD 24712 at three different rotational phases. We selected this particular CP2 star because Preston (1972) concluded from measurements of its effective magnetic field, using lines of different elements, that $\mathrm{Eu}, \mathrm{Mg}$ and $\mathrm{Ti}+\mathrm{Cr}+\mathrm{Fe}$ lines give decreasing values.

\section{Observations and data reduction}

We have carried out $R=100000$ spectroscopic observations of HD 24712 on 1996 October 17-21 with the $1.4 \mathrm{~m}$ Coude Auxiliary Telescope equipped with the Coude Echelle Spectrometer of the European Southern Observatory in the 5412-5456, 5553-5597 and 6122-6172 $\AA$ intervals. The data were reduced by using standard procedures for spectroscopic observations within the NOAO/IRAF package as in Leone \& Catanzaro (1999). After bias subtraction, scattered light subtraction and flat-fielding the normalised final spectra present signal-to-noise $(S / N)$ ratios larger than 200.

On 2001 October 29, 2003 September 7 and 8 we obtained echelle spectra of HD 24712 with the high resolution spectrograph (SARG) (Gratton et al. 2001) equipped with the polarimeter (Leone et al. 2003) on the 3.55-m Telescopio Nazionale Galileo (TNG) (Bortoletto et al. 1998) at the Observatorio del Roque de los Muchachos (La Palma, Spain). In order to measure the effective magnetic field from Stokes $I$ and $V$ line profiles (Mathys 1994), we used a K-prism as $\lambda / 4$ retarder and a Savart plate as beam displacer. Two spectra at the two different positions $\left( \pm 45^{\circ}\right)$ of the K-prism with respect to the Savart plate were obtained in the region from about 4700 to $7000 \AA$ with a resolution of $R=115000$.
Stokes $V$ parameters were measured considering that the following equations are valid for the emerging $o$-rdinary and $e$-xtraordinary beams:

$$
\begin{aligned}
& s_{+45, \mathrm{o}}=0.5(I+V) G_{\mathrm{o}} F_{+45} \\
& s_{+45, \mathrm{e}}=0.5(I-V) G_{\mathrm{e}} F_{+45} \\
& s_{-45, \mathrm{o}}=0.5(I-V) G_{\mathrm{o}} F_{-45} \\
& s_{-45, \mathrm{e}}=0.5(I+V) G_{\mathrm{e}} F_{-45}
\end{aligned}
$$

where $G$ is the time independent (instrumental) sensitivity at the given wavelength and $F$ is the time dependent sensitivity, for example due to variation of sky transparency and slit illumination. Hence:
$\frac{V}{I}=\frac{R-1}{R+1}$
with $\quad R^{2}=\frac{s_{45, \mathrm{o}} / s_{45, \mathrm{e}}}{s_{-45, \mathrm{o}} / s_{-45, \mathrm{e}}}$.

To measure the circular polarisation, the above formulae were applied to the observed spectra by means of the NOAO/IRAF package after bias subtraction, scattered light subtraction and order extraction. Wavelength calibration was performed for both the ordinary and extraordinary spectra for each frame. A high internal accuracy $\left(10^{-4}\right)$ of the wavelength calibration is particularly important. In the present paper we only consider wavelength intervals where $S / N$ is greater than 100 .

For a more complete coverage of the rotational phase, here we also used the spectra presented by Leone et al. (2000) and by Balona \& Zima (2002). Moreover we de-archived one UVES and two ELODIE spectra.

\section{HD $24712=$ HR $1217=$ HIP $18339=$ DO Eri}

HD 24712 is a rapidly oscillating Ap star (roAp) with periods of photometric (Kurtz 1980; Kurtz et al. 1989) and radial velocity (Matthews et al. 1988) variations near $6.14 \mathrm{~min}$.

Kurtz \& Marang (1987) found that the rotational period of HD 24712 is $12.4572 \pm 0.0003$ days. On the basis of measurements of the effective magnetic field, Mathys \& Hubrig (1997) suggested that it is 12.4610 days. Further measurements of the effective magnetic field presented by Wade et al. (2000a) and Leone et al. (2000) did not support this longer period.

Preston (1972) found that equivalent widths of spectral lines are variable with the period of the effective magnetic field. The maximum positive field almost coincides with the maximum strength of the europium lines. The amplitude of the line strength variations appears to increase with the atomic number: it is larger for heavy elements, as for example europium, and smaller for iron-group elements. Light elements on the other hand, such as magnesium and sodium, present variations of larger amplitude that are out-of-phase with respect to the rare earths. Bonsack (1979) confirmed the amplitude dependence on the atomic number, pointing out that there are clear discontinuities in the relationship.

A detailed abundance analysis of HD 24712 was performed by Ryabchikova et al. (1997) with the following main results: 1) rare-earth elements are over-abundant with respect to the Sun; 2) cobalt is also over-abundant while the other iron-peak 
elements are under-abundant; 3 ) there is no monotonic correlation between the amplitude of the abundance variations and $Z$; and 4) with the exception of magnesium, rare-earths show the largest amplitudes in their abundance variations.

Ryabchikova et al. (2000) concluded that Co, Nd and Pr are concentrated in one big spot near the visible magnetic pole of the star, while $\mathrm{Mg}$ is depleted in the same region.

\section{Atmospheric parameters}

We have identified the spectral lines of HD 24712 by comparison of the observed spectrum with a SYNTHE (Kurucz \& Avrett 1981) spectrum, assuming an ATLAS9 (Kurucz 1993) atmosphere model with $T_{\text {eff }}=7250 \mathrm{~K}, \log g=4.3$, solar metal opacity scale and microturbulence equal to $1 \mathrm{~km} \mathrm{~s}^{-1}$ as determined by Ryabchikova et al. (1997). Atomic data for the spectral lines have been taken from the Kurucz's lines list published on his web site ${ }^{1}$.

We want to draw attention to spectral lines of high ionisation stages of some rare earth elements which are not expected in our LTE atmosphere model but which are actually present in the spectra of roAp stars. For the atomic data for these lines we refer to Bord (2000) for Nd III and to Dolk et al. (2002) for Pr II and Pr III.

\section{Variability period}

The first estimate of the period of variability of HD 24712 was given by Preston (1972). This author established that the equivalent widths are variable with a 12.448 day period. Bonsack (1979) measured the equivalent widths of the europium lines and suggested a 12.460 day period to avoid a phase shift with respect to Preston's data.

The equivalent widths of europium lines measured by Preston, Bonsack and ourselves (Eu II 6437 and $6645 \AA$ lines) were separately fitted with the sine function:

$W=W_{0}+W_{1} \sin \left(2 \pi \frac{\mathrm{JD}-\mathrm{JD}_{0}}{P}+\Phi\right)$

where JD is the Julian data, $\mathrm{JD}_{0}$ some arbitrary zero point, and $P$ is the period of variability. For any set of data, errors in $W_{0}$, $W_{1}$ and $\Phi$ were estimated from the $\left(\chi_{\min }^{2}+1\right)$ surface (Press et al. 1992). Following Bonsack (1979), we find that assuming the ephemeris (Fig. 1):

$\mathrm{JD}($ Eu max. $)=2440453.66+12.4582 \pm 0.0006$ days

there is no phase shift between the considered data set (i.e. all sets present the same $\Phi$ value) and $\mathrm{JD}_{0}$ was fixed as the instant of the largest observed equivalent widths for europium lines. The error of the period given above was estimated from the most unfavourable combination of $\Phi$ errors. The largest contribution to the error in the period is due to Preston's data whose $\Phi$ error is equal to $0.05 \times 2 \pi$. Lines with constant equivalent width (Fig. 7) testify to the absence of significant systematical differences in the equivalent widths among the different data set.

\footnotetext{
1 http://kurucz.harvard.edu/LINELISTS/GFALL/ gfall. dat
}
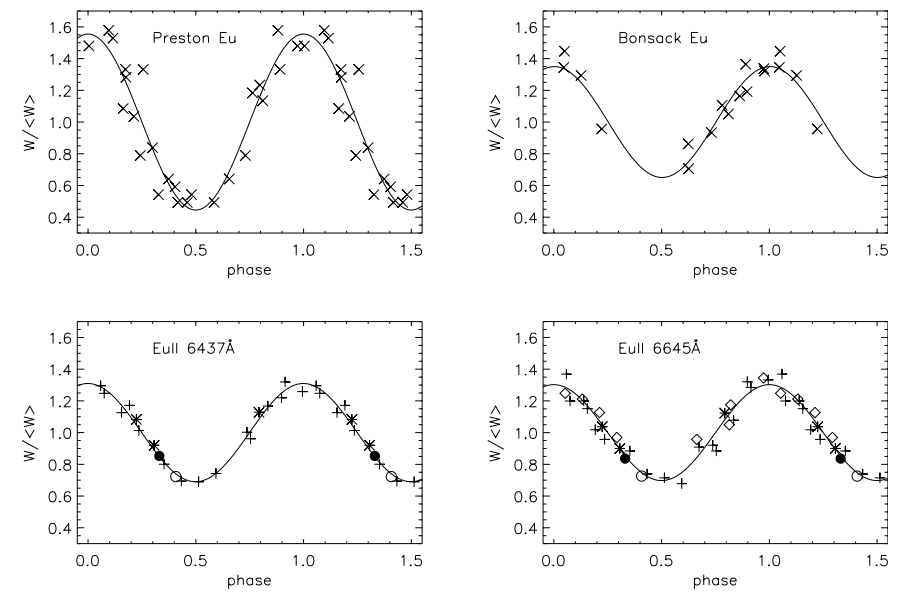

Fig. 1. Equivalent widths of europium lines were used to determine the period of variability of HD 24712. Continuous lines represent a sinusoidal fit to the data. Top left and right panels show Bonsack (1979) and Preston (1972) data respectively. In the bottom panels we display the measurements by Balona \& Zima (2002) (+), OAC (॰), ELODIE (•), TNG $(*)$ and UVES (०) data.

\section{Measuring the effective magnetic field}

Following Mathys (1994), we have computed the first order moment of the Stokes $V$ line profile (valid for unblended spectral lines):

$R_{V}^{(1)}=\frac{1}{W} \int \frac{V_{\mathrm{c}}-V_{\lambda}}{\mathcal{F}_{I_{\mathrm{c}}}}\left(\lambda-\lambda_{I}\right) \mathrm{d} \lambda$

where $W$ is the equivalent width, $V_{\lambda}$ is the flux in Stokes $V$ across the spectral line, $V_{\mathrm{c}}$ the Stokes $V$ flux in the neighbouring continuum, $\mathcal{F}_{I_{\mathrm{c}}}$ the unpolarised continuum flux and $\lambda_{I}$ is the center of gravity of the Stokes $I$ line profile.

$R_{V}^{(1)}$ is related to the effective magnetic field $H_{\mathrm{eff}}$ by

$R_{V}^{(1)}=4.67 \times 10^{-13} g_{\mathrm{eff}} \lambda^{2} H_{\mathrm{eff}}$

measuring the wavelength in $\AA$, where $g_{\text {eff }}$ is the effective Landé factor, $\lambda$ is given in $\AA$ and the field strength is given in Gauss.

Whenever it is possible, Landé factors are from the BASS2000 web site ${ }^{2}$. Otherwise, they are from the VALD database (Kupka et al. 1999). As to the Nd lines, we have adopted the theoretical values given by Bord (2000). Pr III values were kindly supplied by Dr. F. Wyart.

Leone \& Kurtz (2003) found that the effective magnetic field of the roAp stars $\gamma$ Equulei is variable with the oscillation period. To obtain a Stokes $V$ spectrum of HD 24712, we spent $15 \mathrm{~min}$ on the first exposure $+1.67 \mathrm{~min}$ for the CCD reading $+15 \mathrm{~min}$ for the second exposure. The total time, five times longer than the oscillation period (6.14 min) of HD 24712, averages over the oscillations and thus excludes any possible sampling effects due to the pulsations.

Table 3 resumes our results at the three rotational phases in question. We list the average value $\left(H_{\mathrm{eff}}\right)$ and rms scatter of the measurements obtained from $N$ spectral lines of a given

\footnotetext{
${ }^{2}$ http://bass2000.obspm.fr/home.php
} 


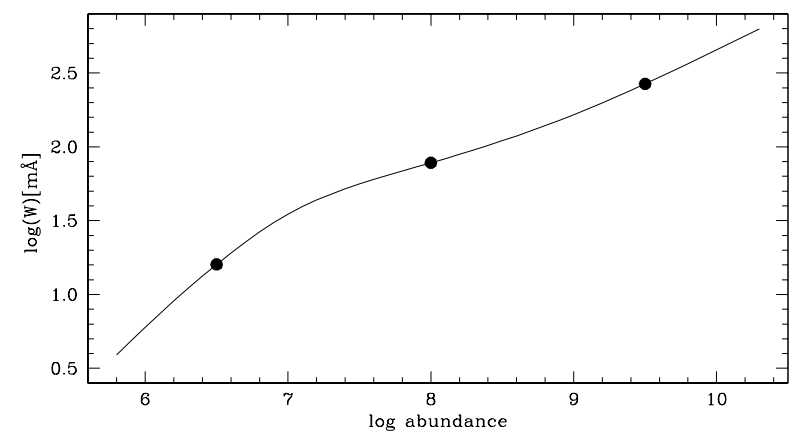

Fig. 2. Curve of growth (for zero magnetic field strength) of the fictitious spectral line. Abundances are relative to a logarithmic hydrogen abundance equal to 12. Dots represent abundances adopted to evaluate the importance of approximated relations in measuring the effective magnetic field, see text.

ion. Averages have been computed assuming a weight equal to the inverse of the uncertainty $\sigma$ in the individual measurements as estimated according to Mathys (1994). In Table 3, we also give the respective averages $\langle\sigma\rangle$ of the $\sigma$ values to show the consistency of uncertainty in measuring the effective field from a single line and scatter within groups of lines of the same ion.

The overall behaviour is characterised by values of the measured effective magnetic field that increase with the atomic number, presenting a maximum at about $Z=60$ (Fig. 3).

Phased with the ephemeris determined in this paper, Fig. 4 shows our $H_{\text {eff }}$ measurements and those by Preston (1972) from $\mathrm{Eu}$ and $\mathrm{Ti}+\mathrm{Cr}+\mathrm{Fe}$ lines. We confirm the difference in the respective fields derived from rare-earths and iron-group elements, and we also verify the $\sim 0.1$ phase difference (Bonsack 1979) of the europium line strength variations with respect to the magnetic field variability (Figs. 1 and 4).

Surprisingly the effective field measured from sodium lines remains always negative. Figure 5 shows the inverted Stokes $V$ profile presented by the Na I $5895 \AA$ line as compared to the Nd II+Nd III $5102 \AA$ and Sm II $5103 \AA$ lines.

The above equation is strictly valid in the weak line limit that implicitly contains the assumptions of a Milne-Eddington solution of the transfer equation for polarised light (Mathys 1989). In order to evaluate how far from the true value measurements of the effective magnetic field can deviate, we have computed the Stokes $I$ and $V$ profiles of a fictitious spectral line (Fig. 2 shows the curve of growth of this fictitious line) by means of the line synthesis code COSSAM, adopting 20 different Zeeman patterns (Stift \& Leone 2003). COSSAM (Stift 2000) solves the equation of transfer for polarised light in the LTE approximation for a plane-parallel atmosphere, including magneto-optical effects. The continuous opacity is interpolated in a wavelength-depth table provided by ATLAS9. The total line opacity is determined by full opacity sampling of the Zeeman sub-components separately. Adopting the atmospheric model for HD 24712 given above, our computations assumed the presence of a uniform magnetic field of 1 or $5 \mathrm{kG}$, forming an angle $\theta=0,35$ or $70^{\circ}$ with respect to the line of sight, together with logarithmic values of the abundance equal to 6.5 , 8.0 and 9.5 relative to a hydrogen logarithmic abundance equal to 12 . Results are summarised in Table 1. With the exception

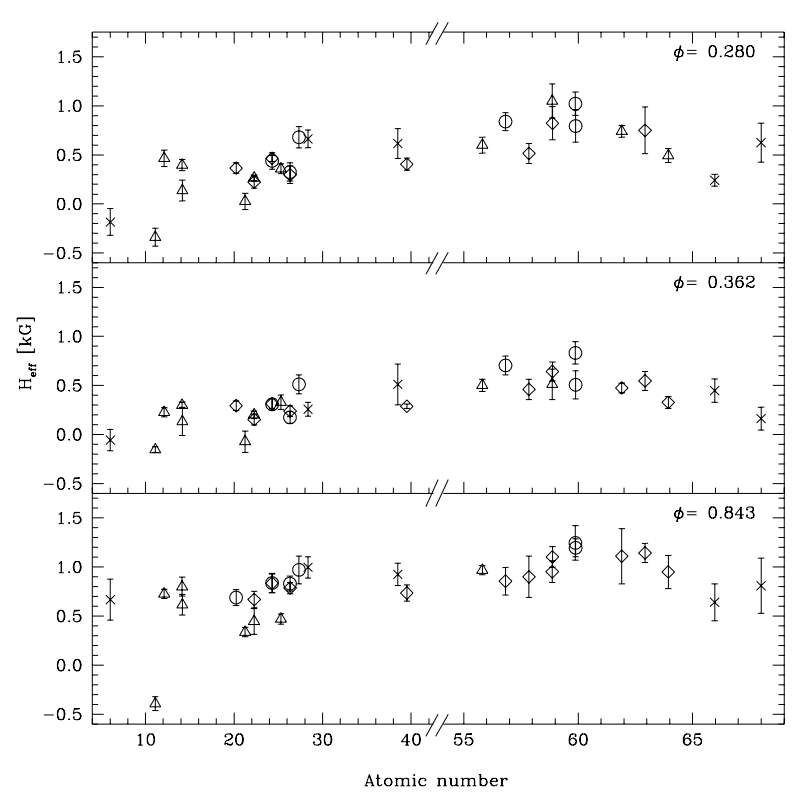

Fig. 3. Measured $H_{\text {eff }}$ value versus atomic number for three rotational phases $(\phi)$. The meaning of the various symbols is related to the number of spectral lines used for the measurement of $H_{\text {eff }}$ : from one line $(\times)$, from two to five lines $(\Delta)$, from six to fifteen lines $(\diamond)$ and from more than fifteen lines $(0)$.
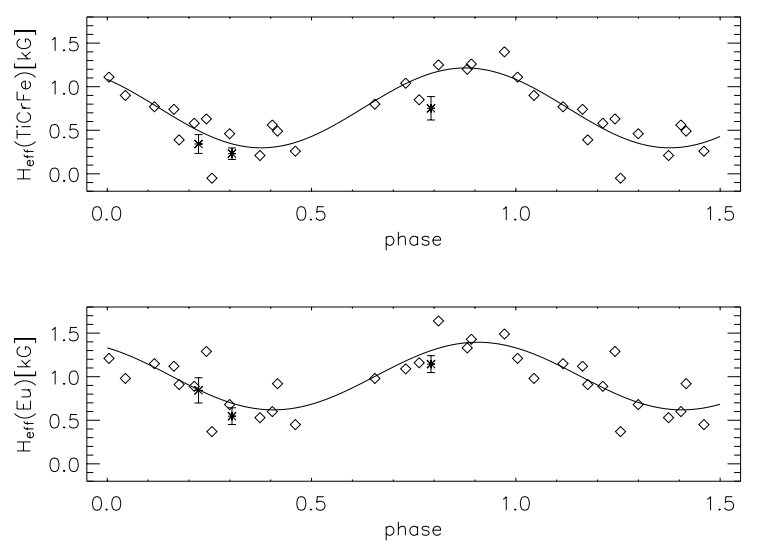

Fig. 4. Variability of the effective magnetic field with the ephemeris determined in this paper. The top panel shows the values obtained by Preston (1972) from $\mathrm{Ti}, \mathrm{Cr}$ and $\mathrm{Fe}$ lines $(\diamond)$ and the values presented here $(*)$. The bottom panel shows the values derived from from Eu lines.

of the ${ }^{2} \mathrm{P}_{3 / 2}-{ }^{2} S_{1 / 2}$ transition, we find that the effective magnetic field measured by using the above relation is, as expected, very close to the input value for weak lines or small $\theta$ angles. In these cases, the standard deviation in measuring the effective magnetic field using a random sample of transitions is of the order of $7 \%$. In the case of strong lines and large $\theta$ angles, we note a general under-estimation of the effective field up to $23 \%$. For a random sample of transitions, we find that that rms can be large up to $18 \%$.

To evaluate the importance of using approximated relations to measure the effective magnetic field in the case of HD 24712, we have assumed an inclination $i=46^{\circ}$ of the rotation axis with respect to the line of sight, an inclination $\beta=30^{\circ}$ of the magnetic dipole axis with respect to the rotation axis and a polar 


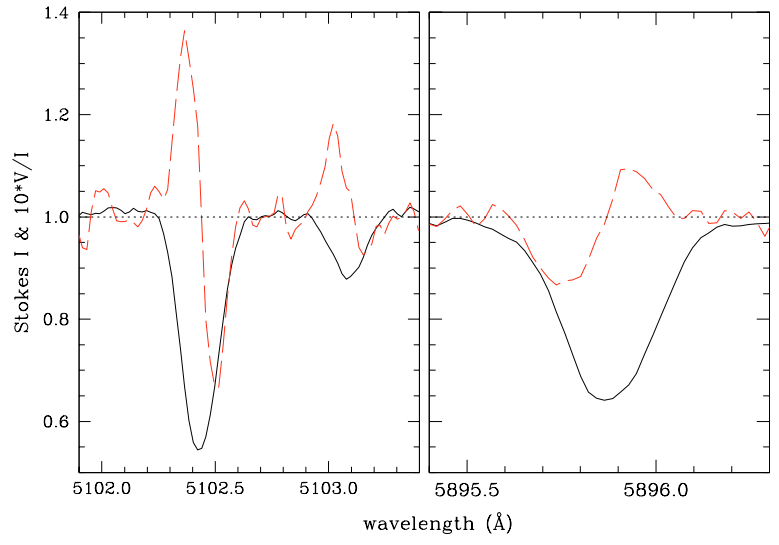

Fig. 5. Stokes $I$ (solid line) and $V / I$ (dashed line) profiles $(\phi=0.362)$ of the Nd II $+\mathrm{Nd}$ II 5102, Sm II 5103 and Na I $5895 \AA$ lines. The Stokes $V / I$ profile of the sodium line is inverted with respect to the other elements indicating that sodium is mainly localised in a negative field region of the stellar disk.
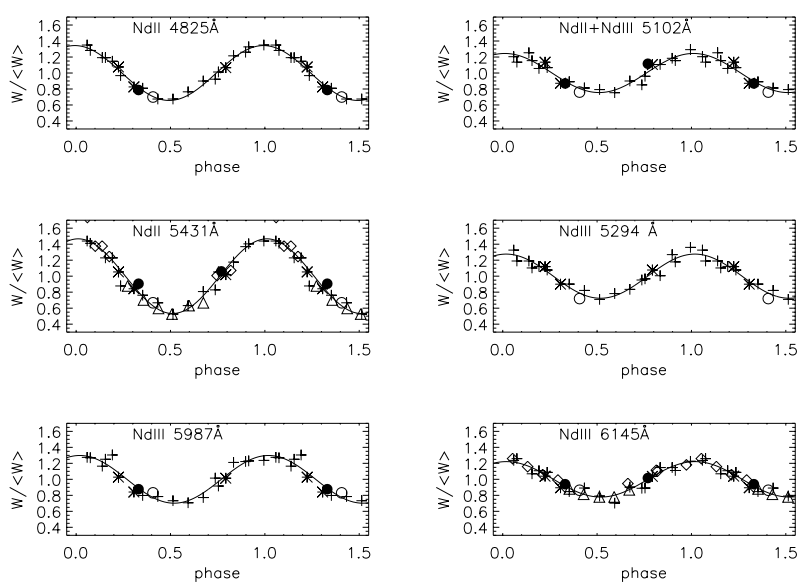

Fig. 6. Equivalent width variability of some $\mathrm{Nd}$ II and $\mathrm{Nd}$ III lines. Symbols are as in Fig. 1 but with CAT data $(\triangle)$ added.

value of the field equal to $4.0 \mathrm{kG}$ (Leone et al. 2000). We have repeated the previous calculations for three values of the rotation phase: $\phi=0$ (when the rotation axis, the dipole axis and the line of sight are in the same plane), 0.25 and 0.5 . We find that for a random sample of transitions, the effective magnetic field is over-estimated up to $6 \%$ if $W$ is in the linear or flat part of the curve of growth. While the field is under-estimated of $9 \%$ for $W$ values in the damping part. The rms values can be as large as $16 \%$ (Table 2).

\section{Variability of spectral lines}

Figures 1, 6-8 show the variability in equivalent width of a number of lines of the elements considered in this paper. The main result is that elements up to iron exhibit an equivalent width variability that is out-of-phase with respect to the effective magnetic field variability (Fig. 4). In contrast, the equivalent widths of elements heavier than iron change in phase with the effective field.

We note that those elements of HD 24712 that Ryabchikova et al. (1997) observed as over-abundant with respect to the sun
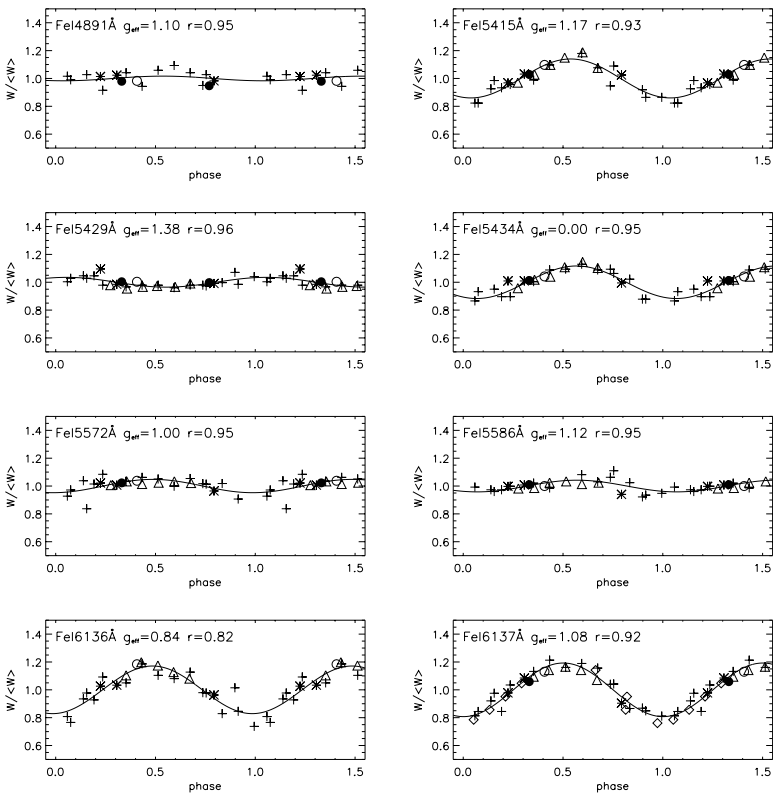

Fig. 7. Equivalent width variability of some Fe r lines. Symbols are as in Figs. 1 and 6. For each line we report the effective Landé factor $\left(g_{\mathrm{eff}}\right)$ and the ratio $(r)$ between the respective equivalent width values computed for $\log \left(\mathrm{Fe} / N_{\text {tot }}\right)=-4.88$ and -4.77 abundance values.

present lines whose equivalent widths change in phase with the effective magnetic field. The changes in the spectral lines of under-abundant elements are out-of-phase.

We do not observe any possible relation between the amplitude $(A)$ of the equivalent width $(W)$ variations and the atomic number (Figs. 1, 6-8). Figure 6 demonstrates how the amplitude variations of neodymium lines do not depend on the ionisation state. We note that the spectral lines of a given element can present $W$ variations with very different $A$ values. As an example, Fig. 7 shows six iron lines with $0 \simeq A \leq 20 \%$. The behaviour of the $\mathrm{Fe}_{\mathrm{I}} 5429 \AA$ line is particular whose equivalent width is slightly variable out-of-phase with respect to the other iron lines. In the limit of the adopted atmosphere model, atomic line list and adopted abundances (Ryabchikova et al. 1997), we find that the differences in $A$ values cannot be ascribed to blending. The most significant blend is expected for the Fe I $5434 \AA$ line whose measured equivalent width could be up to $13 \%$ due to the Co r $5434.566 \AA$.

To check whether such a difference in $A$ can be due to a non-homogeneous distribution of neutral iron on the surface, we have computed - by means of WIDTH9 (Kurucz \& Avrett 1981) - the equivalent widths of the selected six iron lines for the two abundance values derived by Ryabchikova et al. (1997) at the extrema of $H_{\text {eff }}: \log \left(\mathrm{Fe} / N_{\text {tot }}\right)=-4.88$ and -4.77 . The ratio $(r)$ between the equivalent widths obtained for these two values of iron abundance is reported in Fig. 7. We do not find the relation $r \propto A$ as we would expect if a non-homogeneous distribution only were at the origin of the observed equivalent width variability.

For the CP2 star HD 24712, the magnetic intensification of spectral lines (Babcock 1949b; Stift \& Leone 2003) is, in principle, also responsible for the $W$ variations, even for a homogeneous distribution of elements over its surface. In presence of a 
Table 1. For magnetic fields equal to 1 and $5 \mathrm{kG}$, tilted with respect to the line of sight of $\theta$, and three different values of the abundances $(A B)$, we have computed the Stokes $I$ and $V$ profiles for a fictitious line (Fig. 2) assuming 20 different Zeeman patterns. The ratio $r$ between $H_{\text {eff }}$ measured on the basis of Eq. (3), that is strictly valid for weak lines in the Milne-Eddigton approximation, and the input value is reported. The average and rms values for a random sample of transitions are computed. We list also the equivalent width $W$.

\begin{tabular}{|c|c|c|c|c|c|c|c|c|c|c|c|c|c|c|c|c|c|c|c|}
\hline \multirow{3}{*}{$N$} & \multirow{3}{*}{$\begin{array}{l}\text { Transition } \\
B=1 \mathrm{kG}\end{array}$} & \multicolumn{6}{|c|}{$\log A B=6.5$} & \multicolumn{6}{|c|}{$\log A B=8.0$} & \multicolumn{6}{|c|}{$\log A B=9.5$} \\
\hline & & \multicolumn{2}{|c|}{$\theta=0^{\circ}$} & \multicolumn{2}{|c|}{$\theta=35^{\circ}$} & \multicolumn{2}{|c|}{$\theta=70^{\circ}$} & \multicolumn{2}{|c|}{$\theta=0^{\circ}$} & \multicolumn{2}{|c|}{$\theta=35^{\circ}$} & \multicolumn{2}{|c|}{$\theta=70^{\circ}$} & \multicolumn{2}{|c|}{$\theta=0^{\circ}$} & \multicolumn{2}{|c|}{$\theta=35^{\circ}$} & \multicolumn{2}{|c|}{$\theta=70^{\circ}$} \\
\hline & & $\begin{array}{c}W \\
\mathrm{~m} \AA\end{array}$ & $r$ & $\begin{array}{r}W \\
\mathrm{~m} \AA\end{array}$ & $r$ & $\begin{array}{c}W \\
\mathrm{~m} \AA\end{array}$ & $r$ & $\begin{array}{c}W \\
\mathrm{~m} \AA\end{array}$ & $r$ & $\begin{array}{c}W \\
\mathrm{~m} \AA\end{array}$ & $r$ & $\begin{array}{c}W \\
\mathrm{~m} \AA\end{array}$ & $r$ & $\begin{array}{r}W \\
\mathrm{~m} \AA\end{array}$ & $r$ & $\begin{array}{c}W \\
\mathrm{~m} \AA\end{array}$ & $r$ & $\begin{array}{c}W \\
\mathrm{~m} \AA\end{array}$ & $r$ \\
\hline 0 & ${ }^{1} \mathrm{P}_{1}-{ }^{1} \mathrm{P}_{1}$ & 16 & 1.00 & 16 & 1.00 & 16 & 0.99 & 78 & 1.00 & 80 & 0.97 & 82 & 0.94 & 266 & 0.98 & 268 & 0.97 & 269 & 0.98 \\
\hline 1 & ${ }^{2} \mathrm{P}_{3 / 2}-{ }^{2} \mathrm{~S}_{1 / 2}$ & 16 & 0.70 & 16 & 0.69 & 17 & 0.69 & 79 & 0.70 & 81 & 0.67 & 84 & 0.64 & 268 & 0.68 & 270 & 0.67 & 272 & 0.68 \\
\hline 2 & ${ }^{2} \mathrm{G}_{9 / 2}-{ }^{2} \mathrm{~F}_{7 / 2}$ & 16 & 1.00 & 16 & 0.99 & 17 & 0.99 & 78 & 0.99 & 80 & 0.96 & 83 & 0.93 & 267 & 0.97 & 269 & 0.96 & 271 & 0.97 \\
\hline 3 & ${ }^{2} \mathrm{D}_{3 / 2}-{ }^{2} \mathrm{P}_{1 / 2}$ & 16 & 1.00 & 16 & 1.00 & 16 & 1.00 & 78 & 1.00 & 79 & 0.98 & 81 & 0.95 & 268 & 0.97 & 269 & 0.97 & 270 & 0.97 \\
\hline 4 & ${ }^{2} \mathrm{G}_{7} / 2-{ }^{2} \mathrm{~F}_{5 / 2}$ & 16 & 1.00 & 16 & 0.99 & 17 & 0.99 & 78 & 0.99 & 80 & 0.97 & 82 & 0.94 & 268 & 0.97 & 269 & 0.96 & 270 & 0.97 \\
\hline 5 & ${ }^{8} \mathrm{~F}_{7 / 2}{ }^{8} \mathrm{D}_{5 / 2}$ & 16 & 1.00 & 17 & 1.00 & 17 & 0.99 & 82 & 1.03 & 84 & 0.98 & 87 & 0.94 & 270 & 0.99 & 272 & 0.97 & 274 & 0.97 \\
\hline 6 & ${ }^{6} \mathrm{~F}_{5 / 2}-{ }^{6} \mathrm{D}_{3 / 2}$ & 16 & 0.99 & 17 & 0.99 & 17 & 0.98 & 81 & 1.01 & 83 & 0.98 & 85 & 0.94 & 270 & 0.97 & 271 & 0.96 & 273 & 0.96 \\
\hline 7 & ${ }^{2} \mathrm{G}_{7 / 2}-{ }^{2} \mathrm{~F}_{7 / 2}$ & 16 & 0.99 & 17 & 0.99 & 17 & 0.97 & 80 & 0.99 & 83 & 0.92 & 86 & 0.85 & 270 & 0.97 & 272 & 0.94 & 274 & 0.93 \\
\hline 8 & ${ }^{2} \mathrm{D}_{3 / 2}-{ }^{2} \mathrm{P}_{3 / 2}$ & 16 & 1.00 & 17 & 0.99 & 17 & 0.97 & 80 & 0.99 & 83 & 0.92 & 86 & 0.84 & 269 & 0.97 & 272 & 0.94 & 274 & 0.93 \\
\hline 9 & ${ }^{5} \mathrm{D}_{1}-{ }^{5} \mathrm{~F}_{1}$ & 17 & 1.00 & 17 & 0.98 & 17 & 0.95 & 84 & 1.00 & 87 & 0.85 & 91 & 0.70 & 272 & 0.97 & 275 & 0.92 & 278 & 0.89 \\
\hline 10 & ${ }^{3} \mathrm{D}_{2}-{ }^{3} \mathrm{P}_{1}$ & 16 & 1.01 & 16 & 1.00 & 17 & 1.00 & 79 & 1.00 & 81 & 0.97 & 83 & 0.94 & 269 & 0.98 & 270 & 0.97 & 272 & 0.97 \\
\hline 11 & ${ }^{3} \mathrm{D}_{1}-{ }^{3} \mathrm{P}_{1}$ & 17 & 1.00 & 17 & 0.99 & 17 & 0.97 & 81 & 1.00 & 84 & 0.90 & 88 & 0.79 & 270 & 0.98 & 273 & 0.94 & 276 & 0.91 \\
\hline 12 & ${ }^{3} \mathrm{D}_{1}-{ }^{3} \mathrm{D}_{1}$ & 16 & 1.00 & 16 & 1.00 & 16 & 1.00 & 79 & 1.00 & 79 & 0.99 & 80 & 0.97 & 269 & 0.96 & 269 & 0.96 & 270 & 0.96 \\
\hline 13 & ${ }^{5} \mathrm{D}_{2}-{ }^{5} \mathrm{~F}_{2}$ & 17 & 1.00 & 17 & 0.99 & 17 & 0.97 & 81 & 1.00 & 85 & 0.90 & 90 & 0.81 & 271 & 0.98 & 274 & 0.94 & 277 & 0.93 \\
\hline 14 & ${ }^{7} \mathrm{D}_{2}-{ }^{7} \mathrm{~F}_{3}$ & 17 & 1.00 & 17 & 1.00 & 17 & 0.99 & 82 & 1.03 & 84 & 0.99 & 86 & 0.94 & 271 & 0.98 & 273 & 0.96 & 275 & 0.97 \\
\hline 15 & ${ }^{6} \mathrm{D}_{5 / 2}-{ }^{6} \mathrm{~F}_{5 / 2}$ & 17 & 1.00 & 17 & 0.98 & 17 & 0.97 & 81 & 0.99 & 86 & 0.89 & 90 & 0.83 & 271 & 0.97 & 275 & 0.93 & 278 & 0.93 \\
\hline 16 & ${ }^{7} \mathrm{~S}_{3}-{ }^{7} \mathrm{P}_{3}$ & 16 & 1.00 & 17 & 0.99 & 17 & 0.98 & 79 & 1.00 & 86 & 0.90 & 91 & 0.90 & 269 & 0.98 & 275 & 0.94 & 279 & 0.96 \\
\hline 17 & ${ }^{9} \mathrm{H}_{6}-{ }^{9} \mathrm{G}_{5}$ & 16 & 1.00 & 17 & 0.99 & 17 & 0.99 & 80 & 1.00 & 82 & 0.96 & 84 & 0.93 & 270 & 0.97 & 272 & 0.95 & 274 & 0.96 \\
\hline 18 & ${ }^{9} \mathrm{D}_{6}-{ }^{9} \mathrm{~F}_{5}$ & 16 & 1.01 & 17 & 1.00 & 17 & 1.00 & 79 & 1.01 & 84 & 0.92 & 89 & 0.92 & 269 & 0.98 & 274 & 0.95 & 278 & 0.97 \\
\hline 19 & ${ }^{9} \mathrm{I}_{7}-{ }^{9} \mathrm{H}_{8}$ & 17 & 1.10 & 17 & 1.09 & 17 & 1.09 & 82 & 1.09 & 89 & 0.97 & 94 & 0.97 & 271 & 1.08 & 277 & 1.03 & 281 & 1.06 \\
\hline & Average & & 0.99 & & 0.98 & & 0.97 & & 0.98 & & 0.93 & & 0.88 & & 0.97 & & 0.94 & & 0.94 \\
\hline & $\mathrm{rms}$ & & 0.07 & & 0.07 & & 0.07 & & 0.07 & & 0.07 & & 0.09 & & 0.07 & & 0.07 & & 0.07 \\
\hline \multicolumn{20}{|c|}{$B=5 \mathrm{kG}$} \\
\hline 0 & ${ }^{1} \mathrm{P}_{1}-{ }^{1} \mathrm{P}_{1}$ & 16 & 1.00 & 16 & 1.00 & 16 & 0.99 & 78 & 1.00 & 103 & 0.78 & 104 & 0.94 & 266 & 0.98 & 289 & 0.89 & 297 & 0.93 \\
\hline 1 & ${ }^{2} \mathrm{P}_{3 / 2}-{ }^{2} \mathrm{~S}_{1 / 2}$ & 16 & 0.70 & 16 & 0.69 & 17 & 0.69 & 95 & 0.74 & 126 & 0.57 & 129 & 0.67 & 279 & 0.69 & 312 & 0.60 & 324 & 0.63 \\
\hline 2 & ${ }^{2} \mathrm{G}_{9 / 2}-{ }^{2} \mathrm{~F}_{7 / 2}$ & 16 & 1.00 & 16 & 0.99 & 17 & 0.99 & 79 & 1.00 & 105 & 0.78 & 106 & 0.95 & 268 & 0.97 & 293 & 0.87 & 301 & 0.92 \\
\hline 3 & ${ }^{2} \mathrm{D}_{3 / 2}-{ }^{2} \mathrm{P}_{1 / 2}$ & 16 & 1.00 & 16 & 1.00 & 16 & 1.00 & 79 & 1.00 & 100 & 0.80 & 104 & 0.91 & 268 & 0.97 & 286 & 0.90 & 293 & 0.93 \\
\hline 4 & ${ }^{2} \mathrm{G}_{7} / 2-{ }^{2} \mathrm{~F}_{5 / 2}$ & 16 & 1.00 & 16 & 0.99 & 17 & 0.99 & 79 & 0.99 & 102 & 0.78 & 104 & 0.93 & 268 & 0.97 & 289 & 0.88 & 296 & 0.92 \\
\hline 5 & ${ }^{8} \mathrm{~F}_{7 / 2}{ }^{8} \mathrm{D}_{5 / 2}$ & 16 & 1.00 & 17 & 1.00 & 17 & 0.99 & 127 & 1.19 & 154 & 0.95 & 167 & 0.92 & 312 & 1.09 & 352 & 0.90 & 368 & 0.96 \\
\hline 6 & ${ }^{6} \mathrm{~F}_{5 / 2}-{ }^{6} \mathrm{D}_{3 / 2}$ & 16 & 0.99 & 17 & 0.99 & 17 & 0.98 & 118 & 1.18 & 141 & 0.95 & 153 & 0.93 & 302 & 1.06 & 332 & 0.90 & 345 & 0.94 \\
\hline 7 & ${ }^{2} \mathrm{G}_{7 / 2}-{ }^{2} \mathrm{~F}_{7 / 2}$ & 16 & 0.99 & 17 & 0.99 & 17 & 0.97 & 111 & 0.99 & 138 & 0.71 & 152 & 0.67 & 294 & 0.97 & 327 & 0.78 & 339 & 0.79 \\
\hline 8 & ${ }^{2} \mathrm{D}_{3 / 2}-{ }^{2} \mathrm{P}_{3 / 2}$ & 16 & 1.00 & 17 & 0.99 & 17 & 0.97 & 107 & 0.99 & 135 & 0.70 & 150 & 0.67 & 290 & 0.97 & 326 & 0.78 & 338 & 0.78 \\
\hline 9 & ${ }^{5} \mathrm{D}_{1}-{ }^{5} \mathrm{~F}_{1}$ & 17 & 1.00 & 17 & 0.98 & 17 & 0.95 & 123 & 1.00 & 150 & 0.50 & 154 & 0.30 & 309 & 0.97 & 349 & 0.63 & 357 & 0.59 \\
\hline 10 & ${ }^{3} \mathrm{D}_{2}-{ }^{3} \mathrm{P}_{1}$ & 16 & 1.01 & 16 & 1.00 & 17 & 1.00 & 90 & 1.04 & 117 & 0.81 & 121 & 0.93 & 277 & 0.99 & 304 & 0.88 & 314 & 0.93 \\
\hline 11 & ${ }^{3} \mathrm{D}_{1}-{ }^{3} \mathrm{P}_{1}$ & 17 & 1.00 & 17 & 0.99 & 17 & 0.97 & 110 & 1.00 & 139 & 0.64 & 153 & 0.56 & 293 & 0.98 & 333 & 0.73 & 344 & 0.71 \\
\hline 12 & ${ }^{3} \mathrm{D}_{1}-{ }^{3} \mathrm{D}_{1}$ & 16 & 1.00 & 16 & 1.00 & 16 & 1.00 & 79 & 1.00 & 89 & 0.86 & 94 & 0.90 & 269 & 0.96 & 277 & 0.92 & 282 & 0.94 \\
\hline 13 & ${ }^{5} \mathrm{D}_{2}-{ }^{5} \mathrm{~F}_{2}$ & 17 & 1.00 & 17 & 0.99 & 17 & 0.97 & 120 & 1.00 & 150 & 0.72 & 167 & 0.69 & 301 & 0.98 & 348 & 0.75 & 362 & 0.76 \\
\hline 14 & ${ }^{7} \mathrm{D}_{2}-{ }^{7} \mathrm{~F}_{3}$ & 17 & 1.00 & 17 & 1.00 & 17 & 0.99 & 126 & 1.19 & 151 & 0.96 & 165 & 0.93 & 311 & 1.08 & 348 & 0.90 & 364 & 0.95 \\
\hline 15 & ${ }^{6} \mathrm{D}_{5 / 2}-{ }^{6} \mathrm{~F}_{5 / 2}$ & 17 & 1.00 & 17 & 0.98 & 17 & 0.97 & 113 & 0.99 & 147 & 0.73 & 162 & 0.77 & 296 & 0.97 & 350 & 0.75 & 365 & 0.78 \\
\hline 16 & ${ }^{7} \mathrm{~S}_{3}-{ }^{7} \mathrm{P}_{3}$ & 16 & 1.00 & 17 & 0.99 & 17 & 0.98 & 83 & 1.00 & 117 & 0.77 & 114 & 1.01 & 272 & 0.98 & 330 & 0.81 & 337 & 0.89 \\
\hline 17 & ${ }^{9} \mathrm{H}_{6}-{ }^{9} \mathrm{G}_{5}$ & 16 & 1.00 & 17 & 0.99 & 17 & 0.99 & 96 & 1.05 & 125 & 0.81 & 130 & 0.92 & 284 & 0.99 & 315 & 0.86 & 327 & 0.91 \\
\hline 18 & ${ }^{9} \mathrm{D}_{6}-{ }^{9} \mathrm{~F}_{5}$ & 16 & 1.01 & 17 & 1.00 & 17 & 1.00 & 81 & 1.01 & 112 & 0.80 & 109 & 1.04 & 271 & 0.98 & 320 & 0.84 & 328 & 0.91 \\
\hline 19 & ${ }^{9} \mathrm{I}_{7}-{ }^{9} \mathrm{H}_{8}$ & 17 & 1.10 & 17 & 1.09 & 17 & 1.09 & 125 & 1.01 & 164 & 0.80 & 171 & 0.92 & 312 & 1.03 & 374 & 0.81 & 389 & 0.88 \\
\hline & Average & & 0.99 & & 0.98 & & 0.97 & & 1.02 & & 0.77 & & 0.83 & & 0.98 & & 0.82 & & 0.85 \\
\hline & $\mathrm{rms}$ & & 0.07 & & 0.07 & & 0.07 & & 0.09 & & 0.12 & & 0.18 & & 0.07 & & 0.09 & & 0.11 \\
\hline
\end{tabular}


Table 2. Extending the results of Table 1 to HD 24712. We adopt the inclination $i=46^{\circ}$, tilting of the magnetic dipole axis $\beta=30^{\circ}$ and polar strength of the field $H_{\mathrm{p}}=4 \mathrm{kG}$ by Leone et al. (2000). We assumed that when the line of sight is closest at the magnetic pole the rotation phase $\phi=0$.

\begin{tabular}{|c|c|c|c|c|c|c|c|c|c|c|c|c|c|c|c|c|c|c|}
\hline \multirow[b]{3}{*}{$N$} & \multicolumn{6}{|c|}{$\log A B=6.5$} & \multicolumn{6}{|c|}{$\log A B=8.0$} & \multicolumn{6}{|c|}{$\log A B=9.5$} \\
\hline & \multicolumn{2}{|c|}{$\phi=0.00$} & \multicolumn{2}{|c|}{$\phi=0.25$} & \multicolumn{2}{|c|}{$\phi=0.50$} & \multicolumn{2}{|c|}{$\phi=0.00$} & \multicolumn{2}{|c|}{$\phi=0.25$} & \multicolumn{2}{|c|}{$\phi=0.50$} & \multicolumn{2}{|c|}{$\phi=0.00$} & \multicolumn{2}{|c|}{$\phi=0.25$} & \multicolumn{2}{|c|}{$\phi=0.50$} \\
\hline & $\begin{array}{l}W \\
\mathrm{~m} \AA\end{array}$ & $r$ & $\begin{array}{l}W \\
\mathrm{~m} \AA\end{array}$ & $r$ & $\begin{array}{l}W \\
\mathrm{~m} \AA\end{array}$ & $r$ & $\begin{array}{l}W \\
\mathrm{~m} \AA\end{array}$ & $r$ & $\begin{array}{l}W \\
\mathrm{~m} \AA\end{array}$ & $r$ & $\begin{array}{l}W \\
\mathrm{~m} \AA\end{array}$ & $r$ & $\begin{array}{l}W \\
\mathrm{~m} \AA\end{array}$ & $r$ & $\begin{array}{l}W \\
\mathrm{~m} \AA\end{array}$ & $r$ & $\begin{array}{l}W \\
\mathrm{~m} \AA\end{array}$ & $r$ \\
\hline 0 & 18 & 1.05 & 18 & 1.07 & 18 & 1.06 & 88 & 0.99 & 85 & 1.04 & 84 & 1.06 & 251 & 0.99 & 248 & 1.02 & 247 & 1.02 \\
\hline 1 & 19 & 0.73 & 18 & 0.75 & 19 & 0.75 & 100 & 0.72 & 95 & 0.77 & 92 & 0.79 & 266 & 0.67 & 261 & 0.69 & 259 & 0.69 \\
\hline 2 & 18 & 1.04 & 18 & 1.07 & 18 & 1.06 & 90 & 0.98 & 87 & 1.03 & 85 & 1.05 & 257 & 0.95 & 254 & 0.97 & 253 & 0.97 \\
\hline 3 & 18 & 1.05 & 18 & 1.07 & 18 & 1.07 & 85 & 1.01 & 83 & 1.05 & 82 & 1.07 & 253 & 0.96 & 251 & 0.97 & 250 & 0.98 \\
\hline 4 & 18 & 1.04 & 18 & 1.07 & 18 & 1.06 & 87 & 0.99 & 84 & 1.04 & 83 & 1.06 & 255 & 0.95 & 253 & 0.97 & 252 & 0.97 \\
\hline 5 & 20 & 1.06 & 19 & 1.09 & 19 & 1.08 & 116 & 1.19 & 108 & 1.28 & 104 & 1.33 & 286 & 1.01 & 277 & 1.05 & 273 & 1.07 \\
\hline 6 & 19 & 1.05 & 19 & 1.08 & 19 & 1.07 & 108 & 1.19 & 101 & 1.26 & 97 & 1.30 & 276 & 1.00 & 269 & 1.03 & 266 & 1.04 \\
\hline 7 & 19 & 1.02 & 19 & 1.05 & 19 & 1.04 & 108 & 0.90 & 102 & 0.94 & 98 & 0.97 & 275 & 0.88 & 268 & 0.91 & 265 & 0.92 \\
\hline 8 & 19 & 1.02 & 19 & 1.05 & 19 & 1.04 & 108 & 0.88 & 102 & 0.92 & 98 & 0.95 & 274 & 0.88 & 268 & 0.90 & 265 & 0.91 \\
\hline 9 & 20 & 1.01 & 20 & 1.02 & 20 & 1.02 & 121 & 0.65 & 114 & 0.67 & 110 & 0.68 & 288 & 0.74 & 280 & 0.76 & 276 & 0.77 \\
\hline 10 & 18 & 1.06 & 18 & 1.08 & 18 & 1.08 & 94 & 1.03 & 90 & 1.08 & 88 & 1.11 & 262 & 0.96 & 258 & 0.99 & 256 & 0.99 \\
\hline 11 & 20 & 1.02 & 19 & 1.05 & 19 & 1.04 & 112 & 0.80 & 105 & 0.83 & 102 & 0.86 & 278 & 0.84 & 272 & 0.86 & 268 & 0.87 \\
\hline 12 & 18 & 1.05 & 17 & 1.07 & 17 & 1.06 & 79 & 1.07 & 77 & 1.09 & 77 & 1.10 & 248 & 0.94 & 247 & 0.95 & 246 & 0.95 \\
\hline 13 & 20 & 1.03 & 19 & 1.06 & 19 & 1.05 & 117 & 0.88 & 110 & 0.94 & 106 & 0.97 & 285 & 0.87 & 277 & 0.90 & 273 & 0.91 \\
\hline 14 & 19 & 1.06 & 19 & 1.09 & 19 & 1.08 & 113 & 1.20 & 106 & 1.28 & 102 & 1.33 & 282 & 1.00 & 274 & 1.04 & 271 & 1.05 \\
\hline 15 & 20 & 1.03 & 19 & 1.06 & 19 & 1.05 & 117 & 0.90 & 110 & 0.96 & 106 & 0.99 & 286 & 0.86 & 278 & 0.89 & 274 & 0.90 \\
\hline 16 & 19 & 1.04 & 18 & 1.07 & 18 & 1.06 & 101 & 0.97 & 98 & 1.00 & 97 & 1.02 & 278 & 0.90 & 272 & 0.93 & 269 & 0.94 \\
\hline 17 & 19 & 1.05 & 18 & 1.08 & 18 & 1.07 & 98 & 1.03 & 93 & 1.09 & 91 & 1.12 & 266 & 0.93 & 261 & 0.96 & 259 & 0.97 \\
\hline 18 & 18 & 1.05 & 18 & 1.08 & 18 & 1.08 & 98 & 0.99 & 96 & 1.02 & 95 & 1.04 & 272 & 0.93 & 266 & 0.95 & 264 & 0.96 \\
\hline 19 & 20 & 1.15 & 19 & 1.19 & 20 & 1.18 & 124 & 1.03 & 116 & 1.10 & 112 & 1.14 & 292 & 0.99 & 282 & 1.03 & 277 & 1.05 \\
\hline av. & & 1.03 & & 1.06 & & 1.05 & & 0.97 & & 1.02 & & 1.05 & & 0.91 & & 0.94 & & 0.95 \\
\hline $\mathrm{rms}$ & & 0.08 & & 0.08 & & 0.08 & & 0.14 & & 0.16 & & 0.16 & & 0.09 & & 0.09 & & 0.09 \\
\hline
\end{tabular}

dipolar field, we should observe the equivalent widths changing with the magnetic field strength in a roughly sinusoidal way. Assuming that iron is more abundant in a belt around the magnetic equator, we can easily explain the out-of-phase $W$ variation with respect to $H_{\text {eff }}$ of the $g_{\text {eff }}$-null Fe I $5434 \AA$ line. Lines with non-null Landé factors experience the largest magnetic intensification near the magnetic field maximum when the iron abundance is lower. This should result in a reduced amplitude of the $W$ variation. However, we do not observe an increase in amplitude for decreasing values of the Landé factor (Fig. 7).

A quantitative study of the possible dependence of the amplitudes of equivalent width variations on the magnetic intensification of spectral lines is not the primary argument of this paper and will rather be the object of a future investigation.

\section{Conclusion and discussion}

Combining our spectroscopic observations of the magnetic chemically peculiar (CP2) star HD 24712 collected between 1996 and 2001 with data from the literature and from archives, we have found that the equivalent width variations of the europium lines are in phase with the oldest data available (Preston 1972), adopting a $12.4582 \pm 0.0006$ day period.

We have also measured the equivalent widths of representative lines for 24 elements in all the observed ionisation states. Lines of elements up to iron are periodically variable out-of-phase with respect to lines of elements heavier then iron. We find an abrupt 0.5 change of phase in the equivalent width variability for elements heavier than iron.

In general, no relation was found between the amplitudes of the variations and either atomic number or ionisation state.

Under the assumption of a dipolar magnetic field for HD 24712, the phase relation between the respective variations in equivalent width and in $H_{\text {eff }}$ suggest that iron is mainly concentrated in a belt about the magnetic equator. The different amplitudes observed for the equivalent width variations cannot be simply ascribed to a non-homogeneous distribution on the stellar surface.

By means of $R=115000$ circular spectropolarimetry of HD 24712 in the 4700-7000 A range, we have measured the effective magnetic field $\left(H_{\text {eff }}\right)$, the average over the visible disk of the longitudinal field component weighted by the line strength, using all the identified unblended lines of 24 elements at the various observed ionisation states. We found that the measured magnetic field strongly depends on the elements considered, with an overall increase of the measured field with the atomic number, reaching an apparent maximum near $Z=60$. As an example, close to the magnetic minimum $(\phi=0.362)$ we measured $-153 \pm 60 \mathrm{G}$ from 3 neutral sodium lines, $175 \pm 60 \mathrm{G}$ from 35 neutral iron lines and $705 \pm 95$ from 27 neutral lanthanum lines. 
Table 3. Effective magnetic field values of HD 24712 for 30 ions and at 3 rotational phases, measured from $N$ unblended lines; the associated error $\sigma_{H}$ is defined as in Mathys (1994). We report the average value $H_{\text {eff }}$ (in Gauss) and the rms as an error estimate. Whenever $H_{\text {eff }}$ was measured only from one spectral line (listed in the last column) we report $\sigma_{H}$ as estimation of the error. For comparison we report also the average $\langle\sigma\rangle$ values. For any ion, we report the average equivalent width $\langle W\rangle$ of selected lines and the associated standard deviation $\sigma_{W}$.

\begin{tabular}{|c|c|c|c|c|c|c|c|c|c|c|c|c|c|c|c|c|}
\hline \multirow[b]{2}{*}{ Ion } & \multicolumn{5}{|c|}{$\overline{J D D}=2452211.615(\phi=0.843)$} & \multicolumn{5}{|c|}{$\mathrm{JD}=2452889.727(\phi=0.280)$} & \multicolumn{5}{|c|}{$\mathrm{JD}=2452890.748(\phi=0.362)$} & \multirow[b]{2}{*}{$\lambda[\AA]$} \\
\hline & $H_{\text {eff }}$ & Error & $\langle W\rangle \pm \sigma_{W}$ & $N$ & $\left\langle\sigma_{H}\right\rangle$ & $H_{\text {eff }}$ & Error & $\langle W\rangle \pm \sigma_{W}$ & $N$ & $\left\langle\sigma_{H}\right\rangle$ & $H_{\text {eff }}$ & Error & $\langle W\rangle \pm \sigma_{W}$ & $N$ & $\left\langle\sigma_{H}\right\rangle$ & \\
\hline $\mathrm{C}_{\mathrm{I}}$ & 668 & 210 & 17 & & & -184 & 136 & 21 & & & -56 & 110 & 22 & & & 5380.337 \\
\hline $\mathrm{Na}$ & -392 & 70 & $48 \pm 26$ & 3 & 194 & -338 & 91 & $66 \pm 33$ & 3 & 136 & -153 & 30 & $83 \pm 45$ & 3 & 104 & \\
\hline $\mathrm{Mg}_{\mathrm{I}}$ & 726 & 47 & $106 \pm 70$ & 4 & 29 & 466 & 83 & $128 \pm 45$ & 4 & 57 & 228 & 46 & $124 \pm 40$ & 5 & 54 & \\
\hline Si I & 800 & 96 & $33 \pm 6$ & 2 & 124 & 397 & 57 & $25 \pm 6$ & 2 & 104 & 299 & 33 & $31 \pm 8$ & 2 & & \\
\hline Si II & 616 & 106 & $69 \pm 14$ & 3 & 58 & 138 & 107 & $67 \pm 16$ & 2 & 67 & 134 & 143 & $61 \pm 22$ & 3 & 91 & \\
\hline $\mathrm{Ca}$ & 689 & 80 & $53 \pm 18$ & 17 & 78 & 365 & 53 & $46 \pm 15$ & 13 & 62 & 294 & 49 & $55 \pm 17$ & 10 & 51 & \\
\hline $\mathrm{Sc}$ II & 337 & 48 & $22 \pm 11$ & 2 & 74 & 26 & 83 & $32 \pm 14$ & 3 & 66 & -72 & 109 & $32 \pm 14$ & 4 & 72 & \\
\hline $\mathrm{Ti}$ I & 445 & 132 & $22 \pm 3$ & 2 & 215 & 265 & 30 & $24 \pm 14$ & 3 & 156 & 202 & 38 & $24 \pm 14$ & 3 & 95 & \\
\hline Ti II & 669 & 84 & $41 \pm 18$ & 11 & 103 & 224 & 62 & $47 \pm 19$ & 11 & 77 & 154 & 55 & $47 \pm 19$ & 10 & 69 & \\
\hline $\mathrm{Cr}$ & 835 & 95 & $44 \pm 20$ & 14 & 92 & 465 & 30 & $53 \pm 39$ & 12 & 99 & 315 & 52 & $38 \pm 17$ & 11 & 66 & \\
\hline $\mathrm{Cr}_{\text {II }}$ & 837 & 98 & $44 \pm 16$ & 17 & 96 & 439 & 85 & $49 \pm 21$ & 19 & 92 & 308 & 60 & $36 \pm 18$ & 22 & 82 & \\
\hline $\mathrm{Mn}_{\mathrm{I}}$ & 471 & 52 & $28 \pm 8$ & 4 & 194 & 359 & 50 & $37 \pm 10$ & 3 & 134 & 330 & 73 & $32 \pm 8$ & 3 & 134 & \\
\hline $\mathrm{Fe}_{\mathrm{I}}$ & 831 & 76 & $44 \pm 13$ & 27 & 81 & 325 & 93 & $49 \pm 14$ & 28 & 82 & 175 & 60 & $46 \pm 16$ & 35 & 66 & \\
\hline $\mathrm{Fe}_{\text {II }}$ & 786 & 57 & $85 \pm 51$ & 13 & 159 & 298 & 90 & $85 \pm 60$ & 8 & 104 & 240 & 55 & $85 \pm 47$ & 12 & 68 & \\
\hline Co I & 971 & 141 & $34 \pm 17$ & 28 & 159 & 680 & 109 & $30 \pm 14$ & 27 & 141 & 512 & 97 & $26 \pm 11$ & 23 & 130 & \\
\hline Ni I & 997 & 109 & 30 & & & 663 & 90 & 37 & & & 258 & 71 & 34 & & & 4831.169 \\
\hline Sr I & 925 & 113 & 52 & & & 616 & 153 & 40 & & & 511 & 208 & 29 & & & 4607.327 \\
\hline$Y_{\text {II }}$ & 735 & 84 & $69 \pm 25$ & 13 & 71 & 405 & 63 & $69 \pm 31$ & 13 & 72 & 289 & 23 & $63 \pm 26$ & 10 & 60 & \\
\hline $\mathrm{Ba}$ & 968 & 46 & $111 \pm 45$ & 4 & 33 & 599 & 81 & $98 \pm 41$ & 4 & 69 & 501 & 63 & $100 \pm 32$ & 6 & 32 & \\
\hline La I & 856 & 140 & $26 \pm 22$ & 14 & 200 & 840 & 92 & $43 \pm 26$ & 21 & 158 & 705 & 95 & $33 \pm 20$ & 27 & 137 & \\
\hline Ce II & 898 & 209 & $37 \pm 33$ & 5 & 131 & 515 & 102 & $31 \pm 21$ & 9 & 216 & 461 & 104 & $30 \pm 24$ & 5 & 109 & \\
\hline Pr II & 952 & 110 & $16 \pm 5$ & 6 & 253 & 1050 & 173 & $16 \pm 6$ & 3 & 233 & 512 & 156 & $29 \pm 16$ & 4 & 133 & \\
\hline Pr III & 1103 & 104 & $62 \pm 24$ & 5 & 110 & 823 & 169 & $57 \pm 17$ & 6 & 116 & 643 & 98 & $49 \pm 20$ & 5 & 64 & \\
\hline Nd II & 1246 & 175 & $31 \pm 8$ & 18 & 206 & 1022 & 120 & $34 \pm 20$ & 18 & 211 & 832 & 114 & $29 \pm 19$ & 18 & 139 & \\
\hline Nd III & 1196 & 90 & $81 \pm 19$ & 15 & 101 & 794 & 165 & $83 \pm 24$ & 17 & 127 & 507 & 143 & $84 \pm 24$ & 17 & 88 & \\
\hline Sm II & 1110 & 280 & $38 \pm 4$ & 7 & 148 & 740 & 60 & $41 \pm 6$ & 4 & 131 & 475 & 49 & $27 \pm 6$ & 7 & 92 & \\
\hline Eu II & 1144 & 97 & $26 \pm 14$ & 6 & 114 & 750 & 238 & $42 \pm 29$ & 6 & 97 & 547 & 96 & $24 \pm 14$ & 5 & 65 & \\
\hline $\mathrm{Gd}_{\text {II }}$ & 950 & 170 & $22 \pm 5$ & 6 & 156 & 494 & 71 & $28 \pm 17$ & 4 & 155 & 328 & 61 & $18 \pm 6$ & 7 & 188 & \\
\hline Dy II & 641 & 188 & 13 & & & 242 & 61 & 17 & & & 448 & 120 & 16 & & & 4731.849 \\
\hline Er II & 809 & 282 & 23 & & & 625 & 198 & 30 & & & 163 & 116 & 19 & & & 4675.618 \\
\hline
\end{tabular}

For the measurement of the effective magnetic field, we have used Eqs. (2) and (3) that are based on a number of restrictive assumptions such as a Milne-Eddigton atmosphere and the weak line approximation (Mathys 1989). This could lead to a scatter in the magnetic field measurements even if lines from one ion only are used. To better understand this point, we have computed with the Stokes code COSSAM the Stokes $I$ and $V$ profiles for a series of fictitious lines with different Zeeman patterns (Stift \& Leone 2003). It turns out that in very strong lines (that indeed are not very common in the HD 24712 spectrum - Table 3), the field measured by means of Eq. (3) can largely deviate (Table 1) from the input field value. However, for the dipolar magnetic field ascribed to HD 24712 the measured effective field could deviate from the real value by up to $10 \%$ (Table 2).

Taking the lines of a given ion only, we have measured the effective magnetic field and have compared the rms with the accuracy of measurements obtained from individual lines. We do not find any dependency of the measured effective field on line strength and note consistency between the internal scatter of the measures and errors in the individual measurements. We conclude that the differences between the measurements of the effective magnetic field from different elements are indeed real. These differences are much larger than the previous $10 \%$ value and the behaviour of sodium cannot certainly be ascribed to the approximated Eq. (3). The field measured from the lines of this element is negative when the rare-earths give positive values larger than $1 \mathrm{kG}$.

Under the hypothesis of a dipolar magnetic field and elements that are not homogeneously distributed over the stellar surface, we conclude that elements heavier than iron, whose equivalent width maxima almost coincide with the effective magnetic field maximum, are mainly located near the visible positive magnetic pole. Lighter elements seem to be mainly distributed in belts around the magnetic equator. As a consequence, larger values of the effective field obtained from elements concentrated in the visible polar region are simply a consequence of their larger weight in Eq. (1). This could be the 


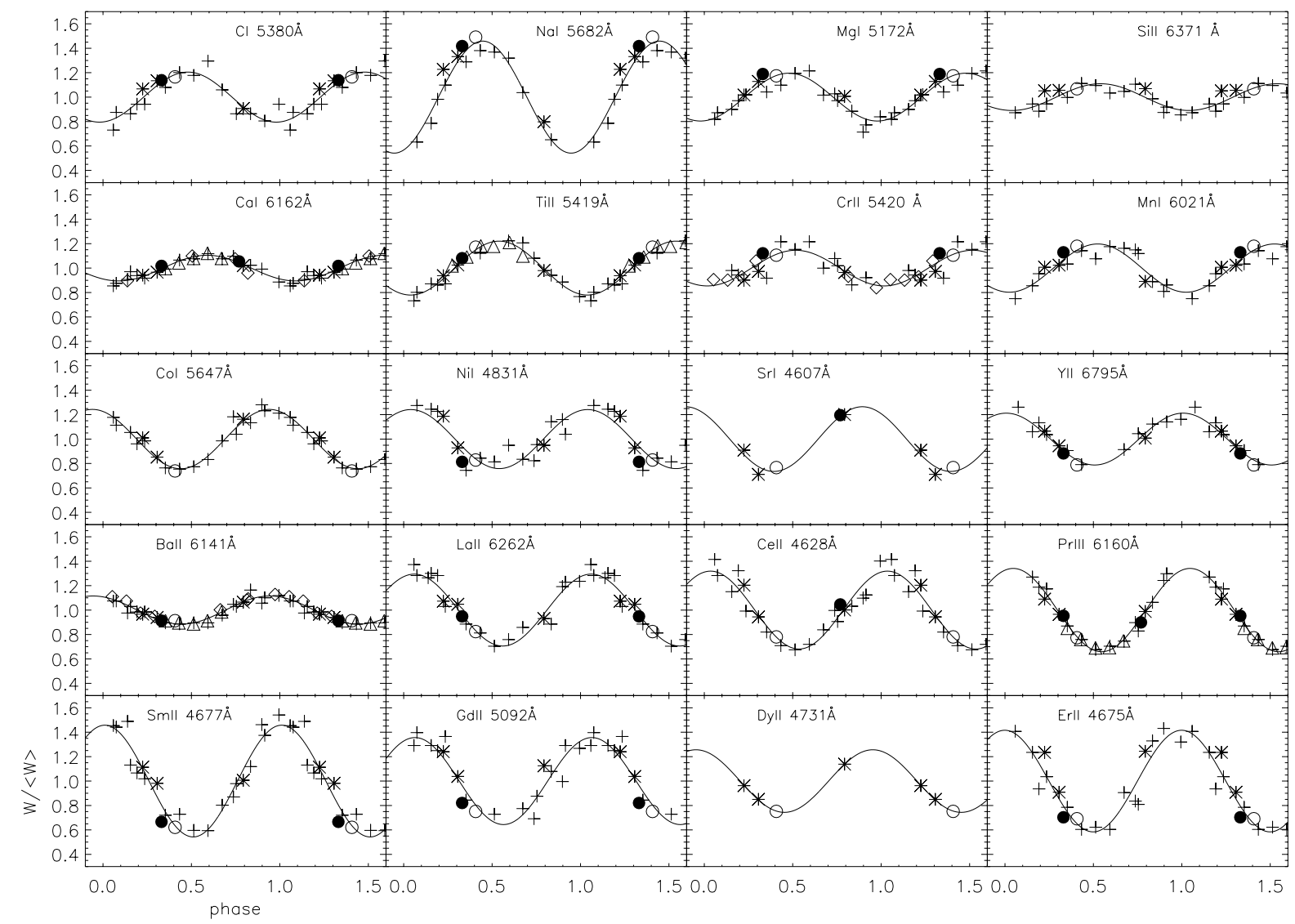

Fig. 8. Variability of the equivalent width of various spectral lines with phase for HD 24712, given in units of their time-average. Continuous lines represent a sinusoidal fit to the data. Symbols are as in Figs. 1 and 6.

case of the rare earths. Lighter elements seem to be mainly distributed in belts around the magnetic equator where the surface magnetic field is weaker.

We conclude that it is not possible to improve the precision by measuring the effective magnetic field of the roAp star HD 24712 with a statistical combination of measurements from lines of different elements. For the elements considered here, effective field measurements obtained from different ionisation states can be considered equal to within the errors.

If we assume that a similar dependence of the measured $H_{\text {eff }}$ values on the elements used applies to all CP2 stars whose elements are not homogeneously distributed over the stellar surface, we conclude that the precision of the final result cannot at priori be improved by combining measurements obtained from different elements. First introduced by Donati et al. (1997) for active stars, the Least-Square Deconvolution (LSD) method combines the signals from different spectral lines to obtain a very high $S / N$ mean line profile. Recently this method has been also applied to CP2 stars (Wade et al. 2000a) but for the same reasons as before, the LSD method cannot at priori improve the precision if applied to lines of different elements.

The modelling of the surface magnetic field of CP2 stars is mainly based on the simultaneous fitting of the variations with the rotational period of several magnetic field quantities, all of which constitute averages over the visible stellar disk, weighted by the local line strengths. In addition to the already defined $H_{\text {eff }}$, there is the mean magnetic field modulus or surface magnetic field $\left(H_{\mathrm{s}}\right)$, the average of the field modulus, then the mean crossover field (Mathys 1995a) and the mean quadratic field (Mathys 1995b). All of these mean quantities can in principle be affected by a non-homogeneous distribution of the various chemical elements. This introduces further parameters to be determined in the mapping of the surface field geometry and precludes the simple use of average quantities from a mixture of elements.

A recent modelling effort illustrates our point: Wade et al. (2000b) have modelled simultaneously $H_{\text {eff }}$ observations (measured from the $\mathrm{H}_{\beta}$ line and from various metal lines) and $H_{\mathrm{s}} \mathrm{ob}-$ servations derived from the Fe I $6149.258 \AA$ line only. They found that the CP2 star HD 12288 presents a dipolar field and HD 14437 a decentred dipole. We note that if the results obtained in this paper for HD 24712 are valid also for the two stars in question, the mean surface field could be largely underestimated and the resulting magnetic map could change considerably. We therefore conclude that only methods based on the simultaneous fitting of full Stokes I $Q U V$ line profiles - of as many elements as possible - can be expected to correctly map both the magnetic field structure and the element distributions (e.g. Piskunov \& Kochukhov 2002).

Acknowledgements. This work has made use of:

- Observations obtained with the Italian Telescopio Nazionale Galileo (TNG) operated on the island of La Palma by the Centro Galileo Galilei of the INAF (Instituto Nazionale di AstroFisica) at the 
Spanish Observatorio del Roque de los Muchachos of the Instituto de Astrofisica de Canarias.

- Data from the UVES Paranal Observatory Project (ESO DDT Program ID 266.D-5655).

- Spectral data retrieved from the Elodie archive at the Observatoire de Haute-Provence (OHP).

We thank Dr. J.F. Wyarth for having computed the Landé factors of Pr III lines and Dr. L. Balona for having made available his spectra of HD 24712.

\section{References}

Babcock, H. W. 1947, ApJ, 105, 105

Babcock, H. W. 1949a, Observatory, 69, 191

Babcock, H. W. 1949b, ApJ, 110, 126

Balona, L. A., \& Zima, W. 2002, MNRAS, 336, 873

Bonsack, W. K. 1979, PASP, 91, 648

Bord, D. J. 2000, A\&AS, 144, 517

Bortoletto, F., Bonoli, C., D’Alessandro, M., et al. 1998, SPIE, 3352, 91

Deutsch, A. J. 1958, in Electromagnetic Phenomena in Cosmical Physics, ed. B. Lehnert, International Astronomical Union (Cambridge University Press), Proc. IAU Symp., 6, 209

Dolk, L., Wahlgren, G. M., Lundberg, H., et al. 2002, A\&A, 385, 111

Donati, J.-F., Semel, M., Carter, B. D., Rees, D. E., \& Collier Cameron, A. 1997, MNRAS, 291, 658

Gray, D. F. 1992, The observation and analysis of stellar photospheres, second edition (Cambridge University press)

Gratton, R., Bonanno, G., Bruno, P., et al. 2001, Exp. Astron., 12, 107

Kupka, F., Piskunov, N. E., Ryabchikova, T. A., Stempels, H. C., \& Weiss, W. W. 1999, A\&AS, 138, 119

Kurtz, D. W. 1980, MNRAS, 200, 807

Kurtz, D. W., \& Marang, F. 1987, MNRAS, 229, 285

Kurtz, D. W., Matthews, J. M., Martinez, P., et al. 1989, MNRAS, 240, 881

Kurucz, R. L. 1993, in IAU Coll. 138, Peculiar versus normal phenomena in A-type and related stars, ed. M. M. Dworetsky, F. Castelli, \& R. Faraggiana, ASP Conf. Ser., 44, 87
Kurucz, R. L., \& Avrett, E. H. 1981, SAO Special Rep., 39

Kushnig, R., Ryabchikova, T. A., Piskunov, N. E., Weiss, W. W., \& Gelbmann, M. J. 1999, A\&A, 348, 924

Landstreet, J. D., \& Mathys, G. 2000, A\&A, 359, 213

Leone, F., Bruno, P., Calì, A., et al. 2003, SPIE, 4843, 465

Leone, F., \& Kurtz, D. W. 2003, A\&A, 407, L67

Leone, F., \& Catanzaro, G. 1999, A\&A, 343, 273

Leone, F., Catanzaro, G., \& Catalano, S. 2000, A\&A, 355, 315

Mathys, G. 1989, Fundam. Cosmic Physi., 13, 143

Mathys, G. 1991, A\&AS, 89, 121

Mathys, G. 1994, A\&AS, 108, 547

Mathys, G. 1995a, A\&A, 293, 733

Mathys, G. 1995b, A\&A, 293, 746

Mathys, G., \& Hubrig, S. 1997, A\&AS, 124, 475

Matthews, J. M., Wehlau, W. H., Walker, G. A. H., \& Yang, S. 1988, ApJ, 324, 1099

Piskunov, N., Kochukhov, O. 2002, A\&A, 381, 736

Press, W. H., Teukolsky, S. A., Vetterling, W. T., \& Flannery, B. F. 1992, Numerical Recipes, 2nd ed. (Cambridge University Press)

Preston, G. W. 1972, ApJ, 175, 465

Preston, G. W. 1974, ARA\&A, 12, 257

Ryabchikova, T. A., Landstreet, J. D., Gelbmann, M. J., et al. 1997, A\&A, 327, 1137

Ryabchikova, T. A., Piskunov, N., Kochukhov, O., et al. 2002, A\&A, 384, 545

Ryabchikova, T. A., Tsymbal, V. V., Malanushenko, V. P., \& Savanov, I. S. 2000, in Magnetic Fields of Chemically Peculiar and Related Stars, ed. Yu. V. Glagolevskij, \& I. I. Romanyuk, Proc. Int. Meeting at Special Astrophysical Observatory of Russian AS, Moscow, 180

Stift, M. J. 1975, MNRAS, 172, 133

Stift, M. J. 2000, A Pecul. Neslett., 33

Stift, M. J., \& Leone, F. 2003, A\&A, 398, 411

Strasser, S., Landstreet, J. D., \& Mathys, G. 2001, A\&A, 378, 153

Wade, G. A., Donati, J.-F., Landstreet, J. D., \& Shorlin, S. L. S. 2000a, MNRAS, 313, 823

Wade, G. A., Kudryavtsev, D., Romanyuk, I. I., Landstreet, J. D., \& Mathys, G. 2000b, A\&A, 355, 1080 